\title{
PALEOCORRENTES E PALEOGEOGRAFIA NA BACIA DO ARARIPE, NORDESTE DO BRASIL
}

\author{
MARIO LUÍS ASSINE
}

\begin{abstract}
PALEOCURRENTS AND PALEOGEOGRAPHY IN THE ARARIPE BASIN, NORTHEAST BRAZIL. The Araripe basin is composed of four unconformity-bounded sequences: 1) Lower sequence; 2) Jurassic-Neocomian sequence; 3) Aptian-Albian sequence, and 4) Albian-Cenomanian sequence. Each sequence shows different alluvial paleocurrent pattern and bears no relation to the geometry of their areas of occurrence, suggesting that these areas are fragments of distinct and originally larger basins. The main sedimentary flow direction N-NW in the Lower sequence (Cariri Formation) is similar to the flow directions in the Paleozoic units in the neighbouring Tucano-Jatobá and Parnaíba basins. The paleocurrent pattern in the Jurassic-Neocomian sequence (Missão Velha and Abaiara formations) indicates a southward paleoflow towards the Recôncavo-Tucano basin. In both sequences the paleocurrent pattern is constant regardless of the several faults, either cutting through them or running along their boundaries, suggesting that the horst-and-graben style is a basin-modifying tectonic product. The fluvial deposits present at the base of the Aptian-Albian sequence (Barbalha Formation) portray southeast paleoflows towards the Sergipe-Alagoas basin. An opposite direction is suggested for the Albian marine transgression in the Santana Formation. Although the sea level was in a global eustatic rising movement, the Albian-Cenomanian deposits of the Exu Formation record the return to continental conditions with rivers flowing westward in the direction of the Parnaíba basin. Such rearrangement in the continental paleodrainage was a consequence of the uplift of Northeast Brazil during the Albian time.
\end{abstract}

Keywords: Araripe basin, Northeast Brazil, paleocurrents, paleogeography

RESUMO A Bacia do Araripe é constituída por quatro seqüências limitadas por discordâncias. Os padrões de paleocorrentes aluviais são diferentes para cada uma das seqüências e sem relação com a geometria de suas áreas de ocorrência, indicando que as mesmas constituem fragmentos de bacias distintas, originalmente mais extensas. Sentidos de fluxo para norte-noroeste nos sedimentos da Formação Cariri são semelhantes aos da Formação Tacaratu (Bacia de Tucano-Jatobá) e aos do Grupo Serra Grande (Bacia do Parnaíba). Na seqüência juro-, neocomiana as paleocorrentes indicam paleodrenagem para sul, em direção à Bacia do Recôncavo-Tucano. Em ambas as seqüências o padrão de paleocorrentes é constante, não sofrendo nenhuma influência das inúmeras falhas que as seccionam ou definem seus limites de ocorrência, indicando que sua estruturação em horstes e grábens é produto de tectônica deformadora. Sedimentos fluviais da base da seqüência aptiano-albiana (Formação Barbalha) apresentam paleofluxos para sudeste em direção à Bacia de Sergipe-Alagoas. E sugerido um sentido oposto para a ingressão marinha albiana presente na Formacão Santana. Embora o nível do mar estivesse num movimento eustático global ascendente, os sedimentos albiano-cenomanianos da Formação Exu registram um retorno às condições continentais, com rios fluindo para oeste, em direção à Bacia do Parnaíba. Tal rearranjo da paleodrenagem continental foi conseqüência de soerguimento da Região Nordeste oriental a partir do Albiano.

Palavras-chave: Bacia do Araripe, Nordeste do Brasil, paleocorrentes, paleogeografia

\section{INTRODUÇ̃̃O}

As bacias interiores do Nordeste do Brasil constituem um conjunto de pequenas bacias fanerozóicas situadas entre as bacias de Tucano-Jatobá, do Parnaíba e Potiguar (Fig. 1). Embora tenham sido consideradas fragmentos de uma única bacia interior (Cordani et al 1984), suas estratigrafias são particulares indicando histórias geológicas distintas.

De todas, a Bacia do Araripe é a mais extensa e a mais estudada. É também a mais complexa já que é caracterizada por uma evolução poli-histórica (Assine 1990, Brito Neves 1990). Sua estratigrafia é composta por quatro seqüências limitadas por discordâncias (Fig. 2).

As sequências inferior e juro-neocomiana apresentam-se confinadas nas sub-bacias do Cariri e de Serrolândia, sendo fortemente estruturadas por falhas de direções nordeste e leste-oeste (Fig. 3). Tal estruturação foi conseqüência da propagação continente adentro dos eventos tectônicos relacionados à fase rifte das bacias marginais brasileiras.

Os sedimentos aptiano-cenomanianos materializam a sedimentação pós-rifte na Bacia do Araripe. Apresentam-se suborizontais com leve caimento para oeste (Fig. 3). Recobrem em discordância angular as unidades inferiores ou repousam diretamente sobre o embasamento cristalino précambriano/eopaleozóico, o que é comum na porção oeste da bacia.
A arquitetura das quatro seqüências não caracteriza embaciamentos completos, com porções marginais e depocentros definidos. As seqüências constituem o registro fragmentário de quatro bacias histórica e geneticamente distintas (Assine 1992). A distribuição geográfica original das diferentes seqüências era mais ampla, ocorrendo hoje remanescentes isolados em várias pequenas bacias adjacentes situadas entre os lineamentos de Pernambuco e da Paraíba (Fig. 1).

As tentativas de reconstrução paleogeográfica já realizadas para os diferentes intervalos de tempo são bastante preliminares, a maioria de cunho regional (Ghignone 1972, Petri 1987, Machado et al. 1989) e umas poucas enfocando mais especificamente a Bacia do Araripe (Mabesoone \& Tinoco 1973, Lima 1978, Santos 1982, Assine 1990, Medeiros 1990).

Em função da natureza incompleta do registro sedimentar da Bacia do Araripe e do número reduzido de informações de subsuperfície, a análise de paleocorrentes é uma ferramenta de grande potencial para a reconstituição de sua história deposicional.

Tal importância é acentuada porque, à exceção dos sedimentos litorâneos e marinhos da Formação Santana, o preenchimento sedimentar da bacia é caracterizado por sistemas deposicionais continentais, aluviais ou lacustres. 


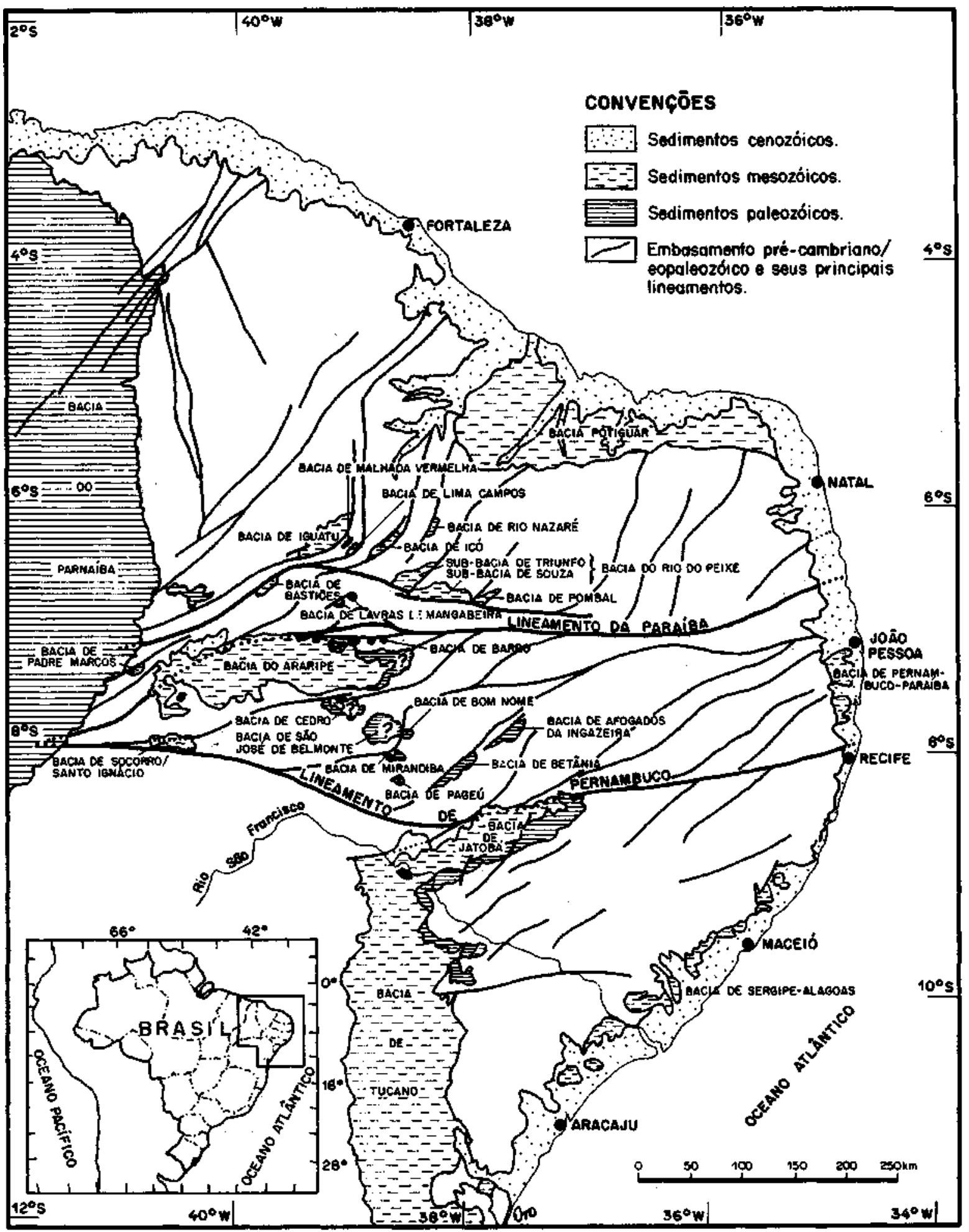

Figura 1 - Bacias fanerozóicas da Região Nordeste do Brasil, contexto onde está inserida a Bacia do Araripe Figure 1 - Phanerozoic basins in Northeast Brazil, showing the Araripe basin

Nestes sistemas o declive topográfico controla o sentido de fluxo das águas em superfície, de forma que as paleocorrentes medidas indicam o mergulho deposicional e a paleodrenagem continental à época da sedimentação das diferentes seqüências.

\section{MÉTODOS E TRABALHOS REALIZADOS As pa-} leocorrentes foram medidas em fácies fluviais a partir do sentido de mergulho dos estratos frontais das estratificações cruzadas tabulares e acanaladas (rank 5 de Miall 1974), por se constituírem as feições indicativas de fluxo mais comumente encontradas na área. Ao todo foram analisadas 37 estações, num total de 622 medidas realizadas.

Sempre que possível procurou-se obter um mínimo de 25 medidas em cada estação, número mínimo recomendado para análises estatísticas (Miall 1990) e suficiente para determinar o vetor médio com um erro máximo de $\pm 30^{\circ}$ (Selley 1982). Este mínimo na maioria dos casos 


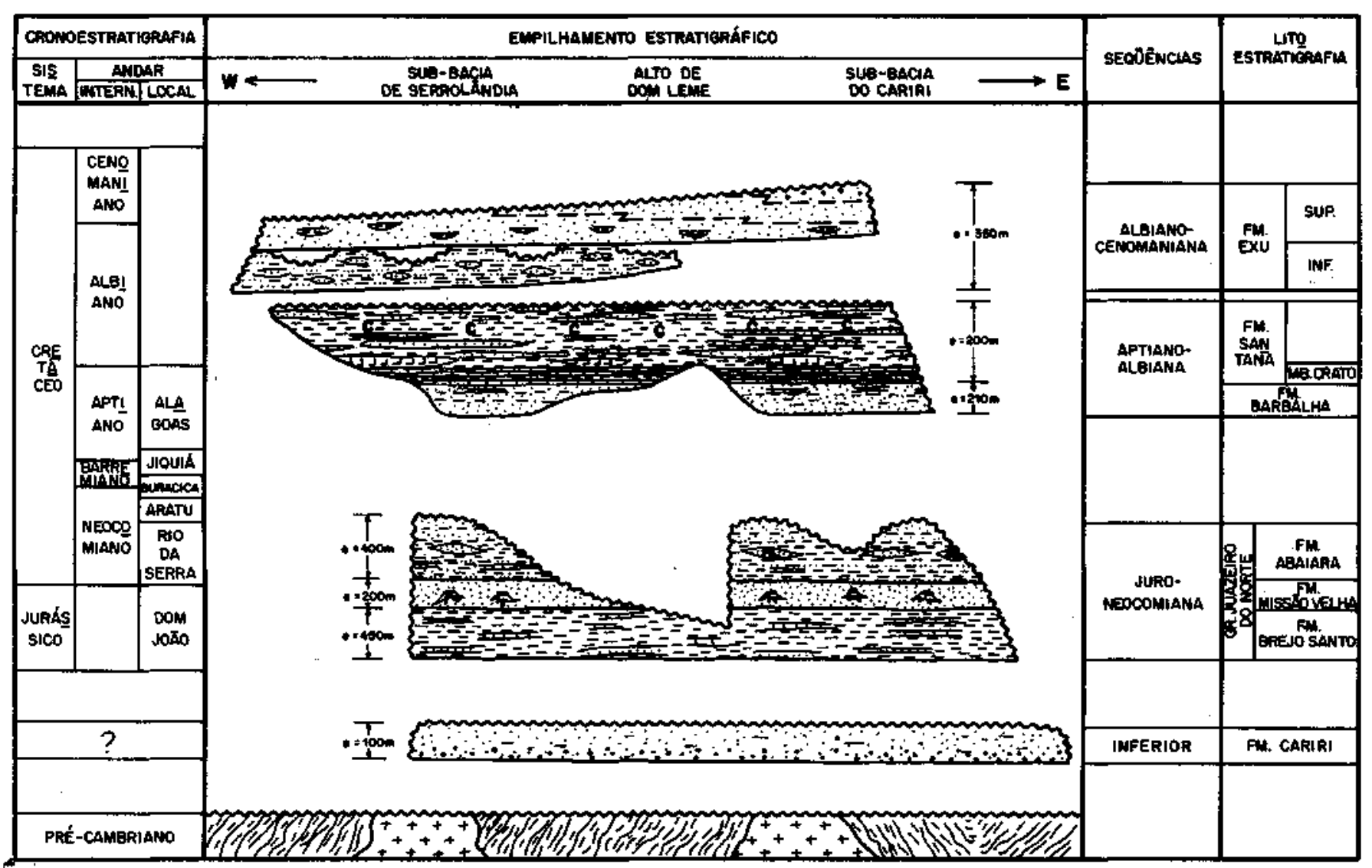

Figura 2 - Coluna estratigráfica da Bacia do Araripe (e = espessura em metros; $C=$ concreções carbonáticas fossilíferas; $\mathrm{G}=$ gipsita; modif. de Assine 1992)

Figure 2- Stratigraphic chart of the Araripe basin ( $\mathrm{e}=$ thickness in meters; $\mathrm{C}=$ fossiliferous carbonate concretions; $\mathrm{G}=$ gypsum; modif. from Assine 1992)

não foi alcançado em função das limitações de exposição e acesso.

Os procedimentos de tratamento e representação dos dados foram os usuais, já consolidados na literatura sobre o assunto (Potter \& Pettijohn 1977, Selley 1982). As medidas ebtidas em afloramentos com mergulho estrutural acima de dez graus foram corrigidas do efeito tectônico através de estereogramas.

Para cada estação, os dados foram distribuídos em classes de $30^{\circ}$, sendo as porcentagens lançadas em histogramas circulares de freqüência (rosetas), que dão idéia visual da dispersão dos valores. A partir dos azimutes medidos $(\alpha)$ e do número de medidas realizadas (n) foram calculados para cada estação o vetor médio $(x)$ e o fator de consistência (f.c.), através das seguintes equações:

$$
\begin{aligned}
& x=\operatorname{arctg}\left(\sum_{\operatorname{sen} \alpha} / \sum \cos \alpha\right) \\
& f . c .=\sqrt{\left(\sum_{\operatorname{sen} \alpha}\right)^{2}+\left(\sum \cos \alpha\right)^{2}} / \mathrm{n}
\end{aligned}
$$

As 37 estações analisadas apresentaram paleocorrentes unimodais, a maioria com alto fator de consistência (f.c. $>$ $0,75)$, mesmo aquelas com menos de 25 medidas.

Todas foram assim agrupadas segundo as quatro diferentes seqüências e apresentadas em mapas individualizados (Figs. 4 e 5). Uma média geral foi calculada para cada sequiência a partir da média aritmética dos vetores médios obtidos em cada estação de medida: seqüência inferior $=352^{\circ}$; sequência juro-neocomiana $=201^{\circ}$ para o Andar Dom João e $169^{\circ}$ para os andares Rio da Serra e Aratu; seqüência aptiano-albiana $=125^{\circ}$; e seqüência albiano-cenomaniana $=256^{\circ}$.

A disposição unimodal das paleocorrentes, o fator de consistência alto e os padrões nitidamente diferentes para cada uma das sequências permitiram uma análise confiável. Basta verificar que a diferença entre as médias gerais de seqüências subseqüentes é maior que o erro máximo de $\pm 30^{\circ}$. Como destacou Miall (1990), a representação de várias estações em mapas pode permitir boas interpretações paleoarribientais, mesmo se o mínimo de 25 medidas por estação não for alcançado.

Com a análise de paleocorrentes buscou-se verificar se as seqüências identificadas na Bacia do Araripe constituem o registro de bacias que ainda mantém sua identidade, ou seja, cujas formas, dimensões e faciologias atuais representam integralmente suas características originais, ou se são fragmentos ou "restos" de bacias (terminologia utilizada por Cordani et al. 1984).

Segundo Selley (1982), a análise de paleocorrentes deduzidas de sedimentos depositados em ambientes controlados pelo mergulho deposicional, tais como os fluviais, permite distinguir bacias sedimentares sindeposicionais das pósdeposicionais ou tectônicas. Em outras palavras, os padrões de paleocorrentes aluviais têm relação direta com as margens e, portanto, com a forma original da bacia. Se as paleocorrentes não apresentarem nenhuma relação com a forma da bacia, sobretudo se os vetores forem paralelos e indiferentes às suas margens, isto indica que se trata apenas de uma fração de uma bacia originalmente maior.

Por fim os padrões de paleocorrentes foram analisados em conjunto com outros dados geológicos a fim de estabelecer conexões para a interpretação da paleogeografía. 


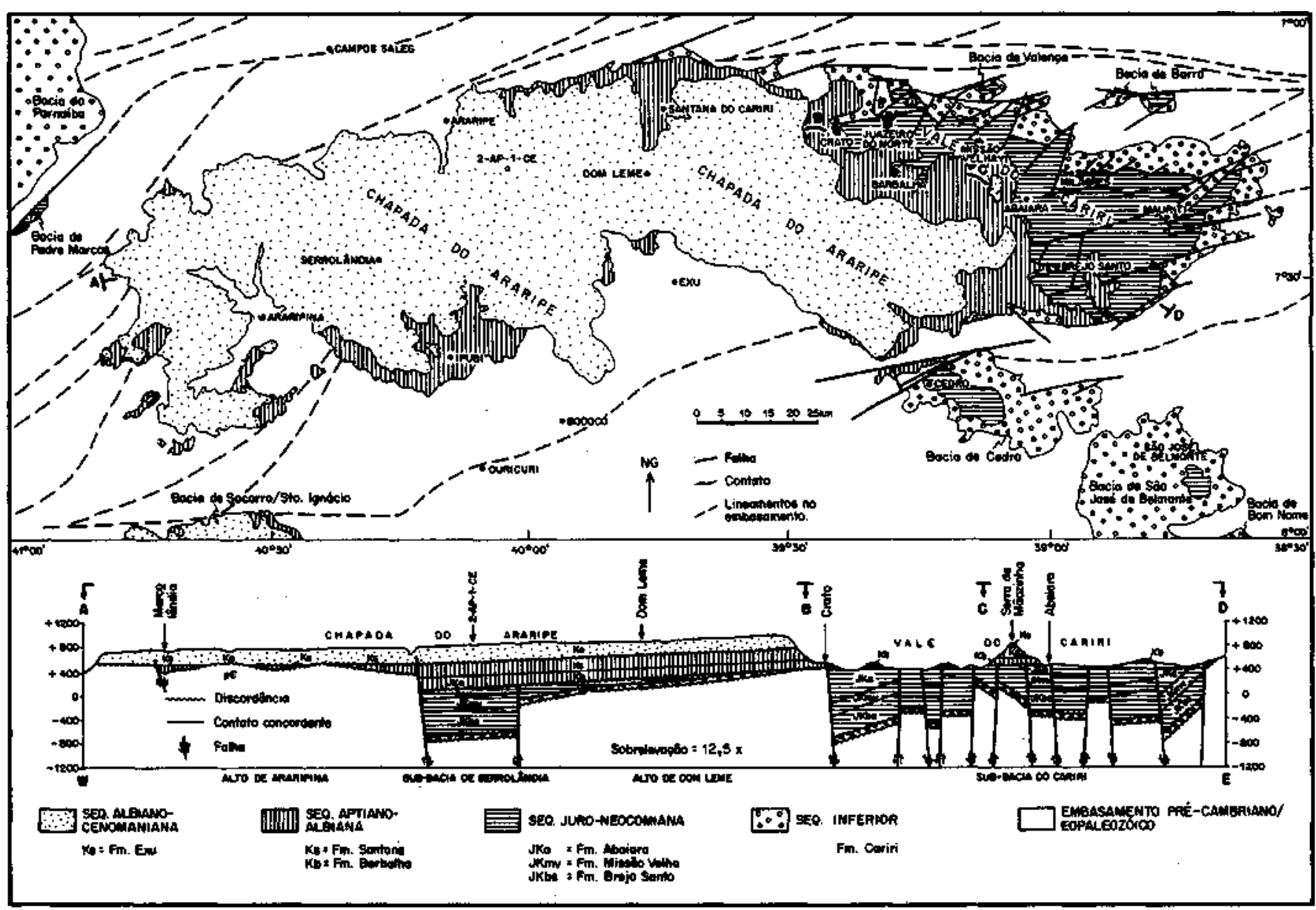

Figure 3-Mapa geológico da Bacia do Araripe, Na seção A-D pode ser observado o padrão estrutural em horstes e grábens das sequências inferior ejuro-neocomiana, afetadas pelo tectonismo do estádio rifte, e a atitude suborizontal das seqüências pós-rifíe (modif. de Assine 1992)

Figure 3 - Geologic map of the Araripe Basin. In the cross-section A-D the horst and graben structural pattern affecting Lower and Jurassic-Neocomian sequences can be observed, as well as the sub-horizontal display of the post-rift sequences (modif. from Assine 1992)

DISCUSSÃO DOS RESULTADOS Como as quatro seqüências são caracterizadas por diferentes padrões de paleocorrentes, sua análise permitiu reconhecer a variação do aporte sedimentar e a modificação das áreas-fonte ao longo do tempo geológico. Permitiu também interpretar movimentações tectônicas e esboçar contextos paleogeográficos a partir da integração com os dados disponíveis na literatura sobre as bacias adjacentes.

Sequência Inferior Os arenitos feldspáticos e/ou conglomeráticos da Formação Cariri, interpretados como produto de sistemas fluviais entrelaçados, ocorrem no Vale do Cariri e em subsuperfície na sub-bacia de Serrolândia (Fig.3). Nove estações analisadas (139 medidas) apresentaram paleocorrentes unimodais e rumo geral de fluxo para norte-noroeste (Fig. 4A), com média geral indicando mergulho deposicional para o azimute $352^{\circ}$.

Por ser considerada afossilífera e em função da similaridade litológica com a Formação Tacaratu (Bacia de Tucano-Jatobá), Braun (1966) atribuiu idade paleozóica para a Formação Cariri, assim como para unidades semelhantes das bacias de Cedro, São José do Belmonte, Mirandiba, Betânia e Afogados da Ingazeira (Fig. 1). Interpretou-as como testemunhos de uma bacia de maior extensão.

Na mesma linha de raciocínio, Ghignone (1972) considerou tais unidades correlatas ao Grupo Serra Grande, advo- gando que originalmente fariam parte da Bacia do Parnaíba, que no Siluriano se estendia muito além do seu atual limite oriental. Esta concepção foi posteriormente adotada por diversos autores, entre os quais Caputo \& Crowell (1985) e Assine (1992).

A similaridade das paleocorrentes destas 'unidades ajustam-se à esta reconstrução paleogeográfica, sugerindo uma paleodrenagem que fluía para noroeste, dos limites orientais do Estado da Bahia aos confins ocidentais do Estado do Maranhão (Fig. 6A).

Carvalho et al. (1994) questionaram, entretanto, a atribuição de uma idade paleozóica para a Formação Cariri, relatando a ocorrência de sete pegadas de dinossauros em sedimentos da unidade. Posicionaram-na no Cretáceo Inferior, sugerindo que tenha se formado no mesmo contexto geológico-temporal das bacias do Rio do Peixe e de Iguatu.

Tal comunicação ainda carece de maiores detalhes, especialmente com relação às rochas onde foram encontradas as pegadas e à sua associação com os andares Rio da Serra/ Aratu da fase rifte daquelas bacias. De qualquer forma, interpretar a Formação Cariri como produto da fase rifte gera uma contradição, já que a mesma ocorre sotoposta à Formação Brejo Santo, portadora de ostracodes típicos do Andar Dom João (Braun 1966, Silva-Telles et al 1991 apud Ponte 1992) e relacionada à fase pré-rifte.

De outra parte, o padrão de paleocorrentes da Formação Cariri, notavelmente constante ao longo da bacia, indica que 
a sedimentação não teve relação alguma com a geometria dos sítios em que hoje os sedimentos estão dispostos. As ocorrências representam restos de uma bacia mais ampla, preservados da erosão por estarem alojados em grábens ou depressões do embasamento, ou ainda devido à intensa silicificação.

O padrão paralelo para norte-noroeste dos vetores médios de paleocorrentes na Formação Cariri, que independe da
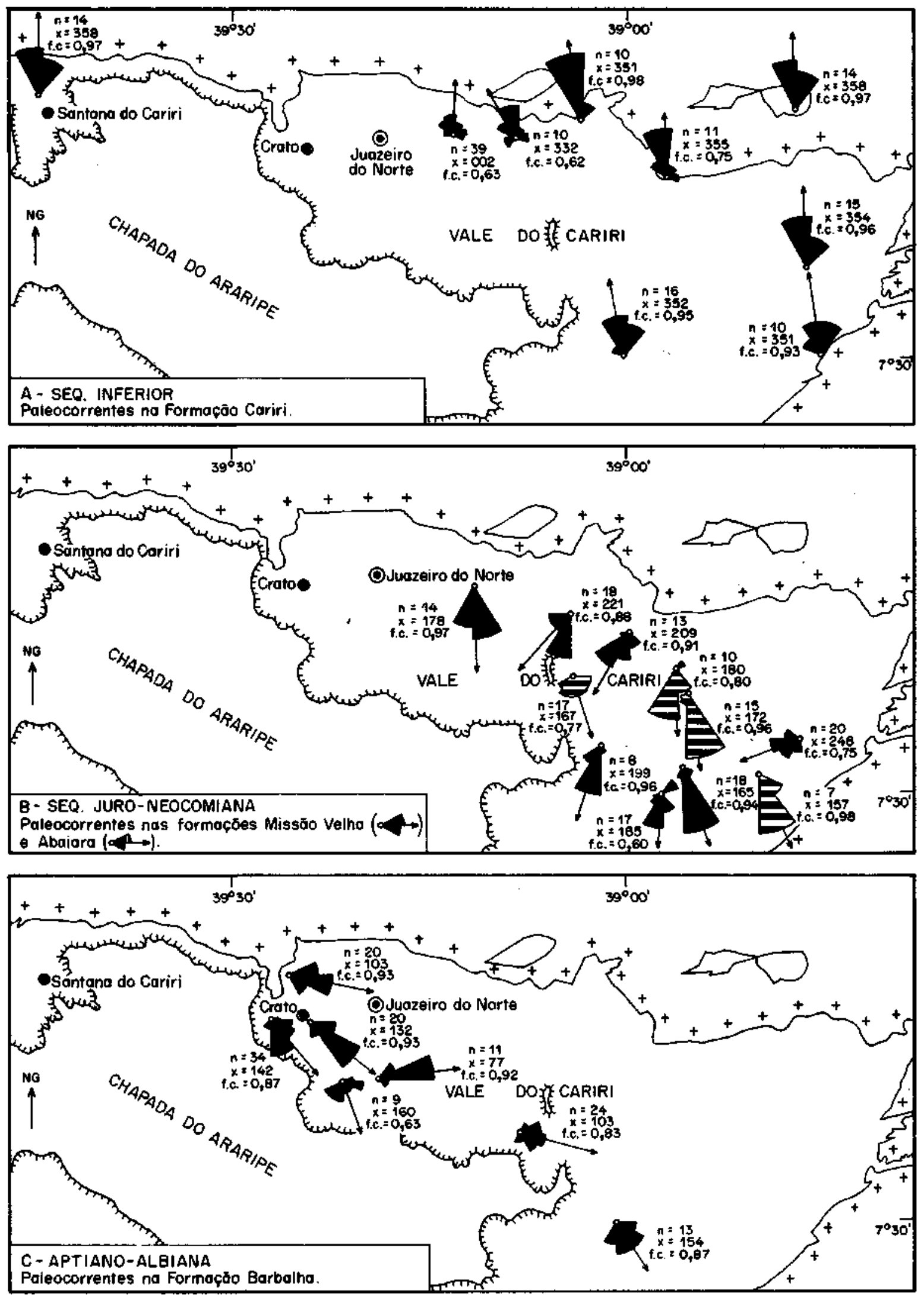

Figura 4 - Mapas de paleocorrentes das sequências inferior, juro-neocomiana e aptiano-albiana

Figure 4 - Paleocurrent maps of the Lower, Jurassic-Neocomian and Aptian-Albian sequences 


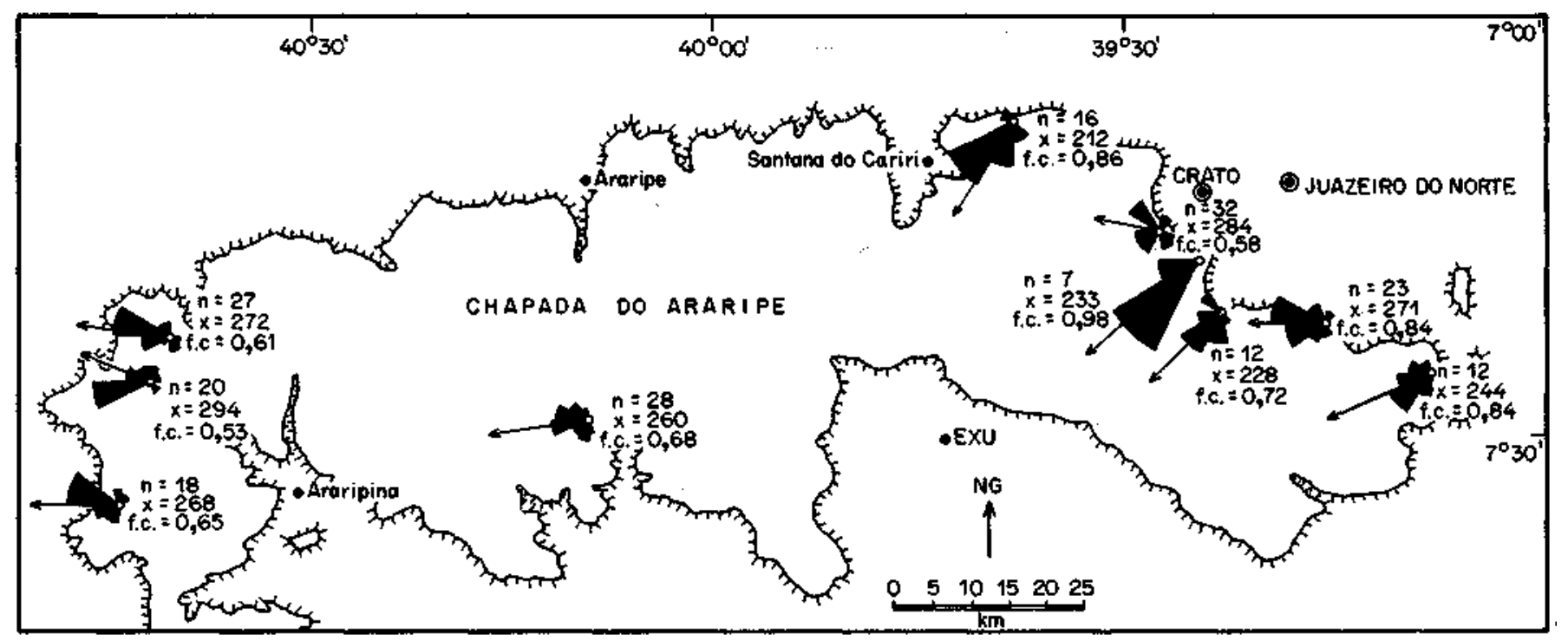

Figura 5-Mapa de paleocorrentes da seqüencia albiano-cenomaniana

Figure 5 - Paleocurrent map of the Albian-Cenomanian sequence

geometria e das falhas limítrofes das áreas de ocorrência da unidade (Fig. 4A), não apresenta similaridades com os padrões da fase rifte. Nas bacias de Iguatu e Rio do Peixe, por exemplo, a paleodrenagem é de natureza centrípeta (Mabesoone et al. 1979) e ajustada às bordas das bacias, definidas por falhamentos sindeposicionais.

Além disso, a faciologia das bacias de Iguatu e Rio do Peixe evidencia sedimentação intimamente associada à movimentação tectônica ao longo dos lineamentos do embasamento pré-cambriano/eopaleozóico (Ghignone et al. 1986, Françolim \& Szatmari 1987), inclusive com a presença de fanglomerados interdigitados com lamitos lacustres. Esta característica da fase rifte contrasta com a monotonia litológica da Formação Cariri, cuja faciologia não sofre mudanças nas proximidades das inumeras falhas que lhe seccionam, indicando que se tratam de falhas posteriores à sedimentação, produto de tectônica deformadora.

Se uma idade Mesozóica for confirmada para a Formação Cariri, uma nova interpretação deve ser buscada. Uma linha de raciocínio a ser investigada é a sua possível correlação com as unidades do Grupo Lavras da Mangabeira, de idade jurássica (Ponte 1994b).

Sequência Juro-Neocomiana A seqüência juroneocomiana da Bacia do Araripe representa a sedimentação contemporânea à Reativação Wealdeniana, esta caracterizada por um conjunto de eventos tafrogênicos de rifteamento continental que promoveu a fragmentação do Gondwana e a abertura do Atlântico sul.

ANDAR DOM JOÂO O Andar Dom João caracterizou-se como um período de estiramento litosférico viscoelástico com incipinte tectonismo rúptil, que tem sido referido como fase pré-rifte (sin-rifte I de Chang et al. 1988). Tal processo foi responsável pela formação da Depressão AfroBrasileira, uma imensa área topograficamente deprimida e orientada na direção norte-sul (Ponte 1972).

A distribuição geográfica dos sedimentos do Andar sugere que a depressã̃o, do sul da Bahia para norte tenha se bifurcado na altura da cidade de Salvador em dois ramos, prenunciando o rifteamento no Recôncavo-Tucano e em Sergipe-Alagoas.

Na Bacia do Araripe o Andar Dom João está representado pelas formações Brejo Santo e Missão Velha, que a partir do trabalho de Braun (1966) têm sido consideradas correlatas respectivamente às formações Aliança e Sergi da Bacia do Recôncavo-Tucano.

O limite original norte da Depressão Afro-Brasileira não é conhecido, mas até o presente não foram constatados sedimentos do Andar nas bacias de Iguatu, Rio do Peixe e Potiguar. A correlação temporal da Formação Antenor Navarro da Bacia do Rio do Peixe com as formações Missão Velha, Sergi e Serraria (Bacia de Sergipe-Alagoas), aventada por Garcia (1991), não encontra suporte nos dados paleontológicos que posicionam a unidade nos andares Rio da Serra/Aratu (Lima \& Coelho 1987).

A julgar pela área de ocorrência comprovada, conclui-se que seu limite norte foi condicionado pelas estruturas lesteoeste do embasamento pré-cambriano/eopaleozóico que compõe o Lineamento da Paraíba. Não sendo submetido ao estiramento litosférico o bloco a norte permaneceu topograficamente mais elevado, funcionando como área-fonte para as bacias que se formavam a sul.

Esta interpretação coaduna-se com as paleocorrentes medidas na Bacia do Araripe. O padrão de dispersão sedimentar da Formação Missão Velha, deduzido de sete estações analisadas (108 medidas), era para sul e caracterizado por paleocorrentes unimodais com grande média para o azimute $201^{\circ}$ (Fig. 4B). Considerando a abundância de troncos silicificados na Formação Missão Velha, as áreas-fonte a norte do Lineamento da Paraíba deveriam ser cobertas por extensas florestas de coníferas (Garcia 1991).

O paralelismo dos vetores médios de paleocorrentes, os limites definidos por falha, a não-constatação de fácies marginais e a ausência de um padrão definido de variação geográfica de fácies indicam que os sedimentos da Formação Missão Velha constituem apenas fração de uma bacia originalmente mais extensa, que possivelmente integrava uma rede hidrográfica que fluía para sul em direção à Bacia do Recôncavo-Tucano (Fig. 6B). Provavelmente, entretanto, não constituíam uma mesma bacia sedimentar, já que texturalmente a seção psamítica da Formação Missão Velha não é significativamente mais grossa e imatura do que a da Formação Sergi, o que seria de se esperar se estivessem condicionadas pelo mesmo nível de base.

ANDARES RIO DA SERRA E ARATU No Neocomiano teve início a fase rifte propriamente dita, ocorrendo o 

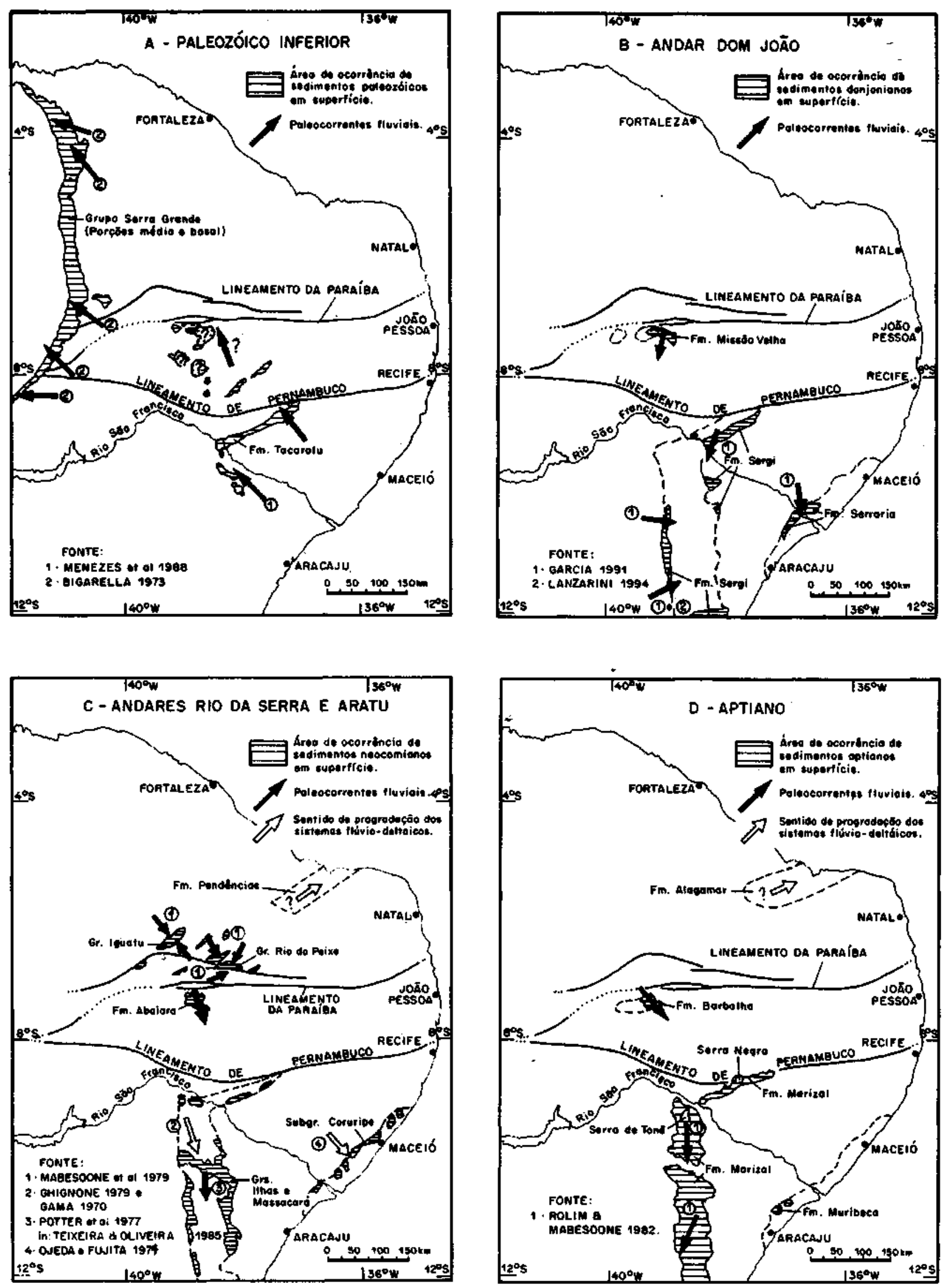

Figura 6 - Esboços da paleodrenagem continental no interior da Região Nordeste do Brasil.

Figura 6 - Paleodrainage outline in the central Northeast Region of Brazil 
primeiro pulso tectônico tafrogênico ao tempo dos andares Rio da Serra/Aratu (sin-rifte II de Chang et al 1988). Nas bacias de Sergipe-Alagoas e Recôncavo-Tucano-Jatobá, a sul do Lineamento de Pernambuco, e nas bacias de Iguatu, Rio do Peixe e Potiguar, a norte do Lineamento da Paraíba, desenvolveram-se grábens e meio-grábens com sedimentação continental sintectônica marcada pela presença de fanglomerados adjacentes às bordas de falha e assoreamento longitudinal dos lagos por sistemas fluviais.

Disto resultaram padrões de paleocorrentes longitudinais com contribuição lateral advinda de leques aluviais adjacentes às falhas limítrofes, ou mesmo paleocorrentes centrípetas denotando paleodrenagem endorreica. As paleocorrentes da Formação Antenor Navarro da Bacia de Rio do Peixe exemplificam este fato, já que segundo Mabesoone et al. (1979) "as direções medidas adaptam-se mais ou menos ao relevo atual, o que sugere que a bacia sedimentar não tem (sic) sido muito maior na época de deposição da sua unidade basal".

A Formação Abaiara da Bacia do Araripe foi depositada no Andar Rio da Serra, possivelmente se estendendo também até o Andar Aratu (Assine 1992, Ponte \& Ponte 1992). Devido à falta de bons afloramentos nela foram analisadas apenas quatro estações (49 medidas) que revelaram paleofluxo para sul, com média geral para o azimute $169^{\circ}$ (Fig. 4B)

Embora estes dados sejam insuficientes para estabelecer a relação entre a geometria de ocorrência e o padrão de paleocorrentes, permitem concluir que o cenário paleogeográfico do Andar Dom João não foi significativamente alterado. A Bacia do Araripe, no transcorrer dos andares Rio da Serra e Aratu, continuava a compor com a Bacia do Recôncavo-Tucano uma mesma paleo-rede hidrográfica, com rios fluindo de norte para sul (Fig. 6C). Mergulho deposicional para sul no Neocomiano da Bacia do Recôncavo-Tucano foi interpretado por Ghignone (1979) em função da disposição espacial dos sistemas deposicionais progradantes e da diminuição da fração arenosa naquele sentido.

Ainda que a Formação Abaiara corresponda cronoestratigraficamente aos sedimentos sintectônicos da fase sinrifte II das bacias a norte do Lineamento da Paraíba e a sul do Lineamento de Pernambuco, nela não foram constatadas avidências de tectonismo sinsedimentar, tais como deformações contemporâneas à sedimentação e cunhas elásticas nos blocos baixos de falha. Sua estruturação em horstes e grábens é aparentemente produto de tectônica deformadora relacionada ao segundo pulso tectônico da fase rifte ao final do Neocomiano (sin-rifte III de Chang et al. 1988), do qual não foram até o momento constatados sedimentos contemporâneos na Bacia do Araripe.

Como a Formação Abaiara é a única unidade neocomiana até o momento constatada entre os lineamentos de Pernambuco e da Paraíba, que definem a Zona Transversal do Nordeste (Fig. 1), é provável que este compartimento geotectônico pré-cambriano tenha se comportado no Neocomiano como um bloco mais estável onde a subsidência foi de pequena magnitude. Por ter orientação leste-oeste, interpôs-se à propagação do rifteamento continente adentro, sendo os esforços distensionais aliviados pela movimentação ao longo das estruturas dos lineamentos de Pernambuco e da Paraíba, que assim comportaram-se como falhas transferentes.

É sugestivo que a megazona de cisalhamento que compõe o Lineamento da Paraíba, reativada no Mesozóico, tenha permanecido como área mais elevada durante o Neocomiano, comportando-se como divisor das águas de superfície que fluíam para sul em direção à Bacia do Recôncavo-Tucano e para norte em direção à Bacia Potiguar (Fig. 6C).
Sequência Aptiano-Albiana A seqüência compreende um ciclo transgressivo-regressivo incompleto no topo. Paleocorrentes em sete estações analisadas (131 medidas) na Formação Barbalha, unidade basal que registra a sedimentação fluvial no início do ciclo, revelaram mergulho deposicional para sudeste, com grande média no sentido do azimute $125^{\circ}$ (Fig. 4C).

Paleofluxos similares foram constatados por Rolim \& Mabesoone (1982) na Formação Marizal (Bacia de Tucano), uma unidade lito, crono e geneticamente correlata à Formação Barbalha. Isto indica que os eventos tectônicos da fase rifte não alteraram significativamente a paleodrenagem continental, que a sul do Lineamento da Paraíba continuou a fluir em direção às bacias do Recôncavo-Tucano e, possivelmente, Sergipe-Alagoas (Fig. 6D).

A seqüência aptiano-albiana registra o advento da fase pós-rifte na Bacia do Araripe, caracterizando uma bacia do tipo sinéclise interior. Sua extensão original era maior que sua atual área de ocorrência, sendo testemunho a Bacia de Socorro na fronteira dos estados de Pernambuco e do Piauí e ocorrências identificadas por Ponte (1994a) nas bacias de Cedro e São José do Belmonte (Fig. 3).

Vale ressaltar que sedimentos correlates lito e cronoestratigraficamente ocorrem muito mais a sul como morros testemunhos na Serra de Tona (Rolim 1984) e na Serra Negra (Braun 1966, Mabesoone \& Tinoco 1973), respectivamente nas bacias de Tucano norte e Jatobá, sugerindo uma continuidade muito maior do que tem sido admitido.

No Aptiano superior, com a elevação global do nível do mar, houve uma transgressão marinha generalizada ao longo das bacias marginais brasileiras, atingindo também o interior da Região Nordeste do Brasil. Registros cretáceos marinhos, aí existentes, restringem-se apenas àqueles do Albiano inferior da Formação Santana da Bacia do Araripe.

A reconstrução paleogeográfica neste intervalo de tempo é matéria controversa, tendo sido já aventados três sentidos de ingressão marinha: a partir da Bacia de Sergipe-Alagoas (Beurlen 1971, Mabesoone \& Tinoco 1973), a partir da Bacia Potiguar (Beurlen 1962, Lima 1978, Petri 1987, Machado et al. 1989) e a partir da Bacia do Parnaíba (Braun 1966, Beurlen \& Mabesoone 1969, Medeiros 1990).

Considerando que as paleocorrentes fluviais das formações Barbalha (Bacia do Araripe) e Marizal (Bacia de Tucano norte) indicam paleodrenagem para sul-sudeste e que os rios, em última análise, correm para o mar, o caminho mais natural para a ingressão é justamente no sentido oposto ao da paleodrenagem continental, ou seja, de sul-sudeste para norte-noroeste. Sugere-se que o mar teria ingressado na Bacia do Araripe através das áreas mais baixas, exatamente os vales fluviais, proveniente da Bacia de Sergipe-Alagoas onde os sedimentos albianos são marinhos.

Esta interpretação está em consonância com a seção apresentada na figura 3 , onde é sugestivo um onlap de leste para oeste. Tal constatação encontra suporte nas observações de Santos (1982), para quem, em direção a oeste, progressivamente estão representadas somente as unidades cada vez mais novas, sendo a seção completa da Formação Santana observada apenas a leste.

Na porção oeste da bacia não ocorrem a Formação Barbalha e o Membro Crato da base da Formação Santana, caracterizando um alto na região de Araripina, apropriadamente referido como plataforma por Ponte (1992). Nesta área os sedimentos marinhos/costeiros da parte superior da Formação Santana recobrem diretamente o embasamento, materializando uma superfície de máxima transgressão à qual estão associados os níveis pelíticos com concreções carbonáticas fossilíferas (Fig. 2).

Quando a transgressão aptiano-albiana alcançou a Plataforma de Araripina encontrou um relevo peneplanizado sobre rochas do embasamento pré-Cambriano/eopaleo- 
zóico, já que aí não ocorrem nem mesmo os sedimentos da Formação Cariri, originando uma extensa área em condições de supra-maré onde se formaram extensas jazidas de gipsita.

Sequência Albiano-Cenomaniana Embora o mar tenha continuado a subir durante o Albiano, num movimento eustático positivo que atingiu o clímax no Turoniano, na Bacia do Araripe houve um retorno às condições continentais já no Albiano médio, com a deposição dos sedimentos tipicamente fluviais da Formação Exu (Assine 1990 e 1992, Brito Neves 1990).

Medidas de paleocorrentes apresentaram sentidos de fluxo para oeste-sudoeste (Fig. 5). Nas dez estações analisadas (195 medidas) as paleocorrentes são unimodais com média geral para o azimute $256^{\circ}$. Tais dados caracterizam uma mudança notável em relação ao padrão das paleocorrentes dos sedimentos aptianos.

Deduz-se que o retorno às condições continentais na Bacia do Araripe foi resultado de uma movimentação vertical positiva (soerguimento) do bloco nordestino, já a partir do Albiano médio. Este soerguimento teria sido diferenciado, mais intenso a leste, promovendo uma reestruturação completa da paleodrenagem continental no interior do Nordeste, que passou a fluir para oeste em direção à Bacia do Parnaíba.

A Chapada do Araripe, sustentada pelos arenitos da Formação Exu, representa um relevo residual delimitado por escarpas nitidamente erosivas. Testemunhos de sua outrora maior extensão existem a sudoeste da bacia (Bacia de Socorro/Santo Ignácio) e no Vale do Cariri (Serra da Mãozinha, Fig. 3). Sua amplitude geográfica original era possivelmente muito maior, talvez cobrindo grande parte da porção oriental da Região Nordeste do Brasil, dela restando um único morro-testemunho na Serra Negra (Bacia de Jatobá em Pernambuco), onde ocorrem arenitos correlates em cotas altimétricas similares as da própria Chapada do Araripe (Braun 1966).

Embora haja carência de informações sobre paleocorrentes na Formação Itapecuru, os dados levantados na Bacia do Araripe consubstanciam a reconstituição paleogeográfica de Petri \& Fúlfaro (1983), segundo a qual os sedimentos da Formação Exu constituiriam as porções marginais dos extensos tratos deposicionais aluviais da Formação Itapecuru, nurna ampla paleo-rede hidrográfica que convergia para norte em direção à então recém-formada margem equatorial brasileira (Fig. 7).

CONCLUSÕES As quatro sequências estratigráficas identificadas na Bacia do Araripe apresentam padrões de paleocorrentes fluviais bem definidos e distintos.

Os padrões unimodais constatados e sua constância ao longo da bacia, sem relação alguma com a forma dos sítios de ocorrência de cada seqüência, confirmam que o registro sedimentar da Bacia do Araripe é fragmentário. A distribuição geográfica de cada seqüência é resultado da preservação apenas parcial de bacias outrora mais extensas, sendo a forma determinada por erosão remontante ou embutimento em blocos baixos de falha.

As tentativas de reconstrução paleogeográfica devem levar em consideração as características geológicas das seqüências de bacias adjacentes, com as quais as seqüências identificadas na Bacia do Araripe guardam relação estreita, podendo inclusive ter integrado tratos deposicionais contínuos.

As paleocorrentes da Formação Cariri são similares às de unidades paleozóicas de bacias adjacentes, mas seu posicionamento no Paleozóico, baseado em correlações, foi questionado a partir da descoberta de pegadas atribuídas a dinossauros. Por outro lado, sua associação com a fase rifte é contraposta pela sua faciologia, pelo seu

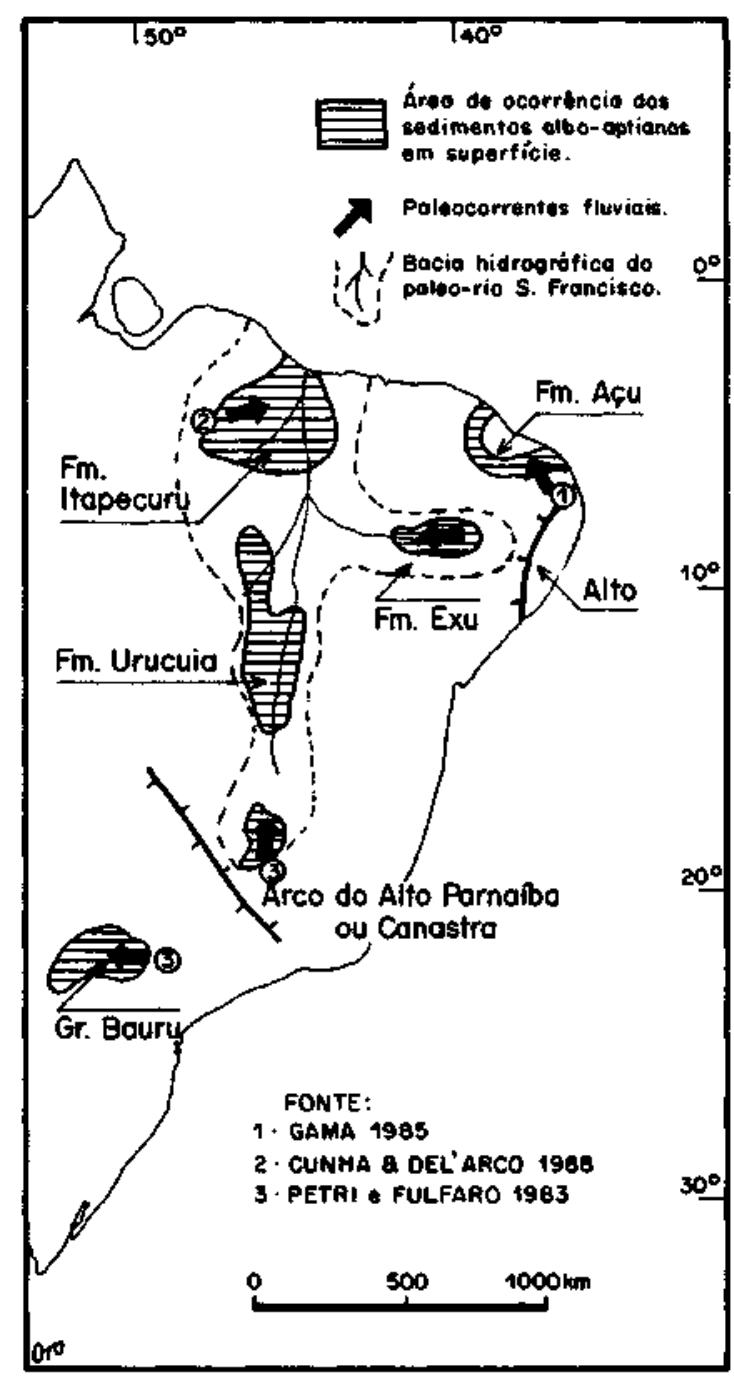

Figura 7 - Esboço da paleodrenagem continental albianocenomaniana na porção oriental do Brasil (modif. de Petri \& Fúlfaro 1983)

Figure 7 - Paleodrainage outline for the Albian-Cenomanian in eastern Brazil (modif. from Petri \& Fúlfaro 1983)

padrão de paleocorrentes e pelo fato de estar sotoposta à Formação Brejo Santo (Andar Dom João). Desta forma, seu papel na evolução da bacia ainda carece de dados mais conclusivos.

As paleocorrentes nas unidades juro-neocomianas da Bacia do Araripe indicam mergulho deposicional para sul, constante na bacia e independente das estruturas e mergulho estrutural dos blocos tectônicos. A Bacia do Araripe integrava uma paleodrenagem continental que fluía para sul em direção à Bacia do Recôncavo-Tucano, mas constituia uma bacia distinta.

A seqüência aptiano-albiana registra o início da fase pósrifte na Bacia do Araripe. A Formação Barbalha correlaciona-se com a Formação Marizal da Bacia do RecôncavoTucano, ambas apresentando paleocorrentes para sul. Um sentido oposto, de sul-sudeste para norte-noroeste, é sugerido para a transgressão aptiano-albiana, que causou uma breve ingressão marinha na Formação Santana da Bacia do Araripe.

O mergulho deposicional da sequência albiano-cenomaniana passou a ser para oeste. Os rios da Formação Exu 
fluíam no sentido da Bacia do Parnaíba, fazendo parte da bacia hidrográfica de um paleo-rio São Francisco. Tal rearranjo na paleodrenagem continental foi interpretado como o resultado de soerguimento da Região Nordeste do Brasil a partir do Albiano médio.
Agradecimentos $\mathrm{O}$ autor externa seus agradecimentos ao desenhista Oto Laurentino Rosa pelas ilustrações; a Dr. Almério Barros França pela colaboração no abstract; e à Petrobrás pelo apoio financeiro para os trabalhos de campo.

\section{REFERÊNCIAS BIBLIOGRÁFICAS}

ASSINE, M. L. 1990. Sedimentação e tectônica da Bacia do Araripe, Nordeste do Brasil. Rio Claro. 124p. (Dissertação de Mestrado, IGCE/UNESP).

ASSINE, M. L. 1992. Análise estratigráfica da Bacia do Araripe, Nordeste do Brasil. Rev. Bras. Geoc., 22(3):289-300.

BEURLEN, K. 1962. A geologia da Chapada do Araripe. An. Acad. bras. $C L, 34(3): 365-370$.

BEURLEN, K. 1971. Bacias sedimentares do bloco brasileiro. Natal, Estudos Sedimentológicos, 1(2):7-32.

BEURLEN, K. \& MABESOONE, J.M. 1969. Bacias cretáceas intracontinentais do Nordeste do Brasil. Campinas, Notícia Geomorfológica, 9(18):19-34.

BIGARELLA, J.J. 1973. Paleocprrentes e deriva continental (comparação entre América do Sul c Africa). Curitiba, B. Paranaense Geoc., 31:141-224

BRAUN, O.P.G. 1966. Estratigrafia dos sedimentos da parte inferior da Região Nordeste do Brasil (bacias do Tucano-Jatobá, Mirandiba e Araripe). Rio de Janeiro, DNPM/DGM, 75p. (Boletim 236).

BRITO NEVES, B.B. 1990. A Bacia do Araripe no contexto geotectônico regional. In: SIMP. SOBRE A BACIA DO ARARIPE E BACIAS INTERIORES DO NORDESTE, 1, 1990, Crato. Atas... Grato, DNPM, p. 21-33.

CAPUTO, M.V. \& CROWELL, J.C. 1985. Migration of glacial centers across Gondwana during Paleozoic Era. Geol. Soc. Am. Bull., 96:1020-1036.

CARVALHO, I. S.; VIANA, M. S. S. \& LIMA, M. E, F. 1994. Dinossauros no Siluriano: um anacronismo crono-geologico nas bacias interiores do NE? In: CONGR. BRAS. GEOL., 38, Camboriú, 1994. B. Resumos Expandidos... Camboriú, SBG, v.3, p.213-214.

CHANG, H.K.; KOWSMANN, R.O. \& FIGUEIREDO, A.M.F. 1988. New concepts on the development of east Brazilian marginal basins. Episodes, 11(3): 194-202.

CORDANI, U.G. \& BRITO-NEVES, B.B. \& FUCK, R.A. \& PORTO, R., THOMAZ FILHO, A., CUNHA, F.M.B. 1984. Estudo preliminar da integração do pré-Cambriano com os eventos íectônicos das bacias sedimentares brasileiras. Rio de Janeiro, PETROBRÁS/ CENPES, 70p. (Série Ciência-Técnica-Petróleo, Seção Explor. Petróleo $\left.\mathrm{n}^{\mathrm{e}} 15\right)$.

CUNHA, B.C.C. \& DEU ARCO, J.0.1988. Variações faciológicas da Formação Itapecuru na região de Santa Inês - MA. In: CONGR. BRÁS. GEOL., 35, Belém, 1988. Anais... Belém, SBG, v.2, p.765-777.

FRANCOLIM, J.B.L. \& SZATMARI, P. 1987. Mecanismo de rifteamento da porção oriental da margem norte brasileira. Rev. Bras. Geoc., 17(2): 196-207.

GAMA, E., Jr. 1970. Modelo geológico das bacias do Recôncavo e Tucano. In: CONGR. BRÁS. GEOL., 24, Brasília, 1970. Anais... Brasília, SBG, p. 191-200.

GAMA, E., Jr. 1985. Estratigrafia genética e classificação global da Bacia Potiguar (RN) e ambiência de petróleo nas bacias mesozóicas do nordeste brasileiro. Rio Claro, 133p. (Tese de Livre-Docência, IGCE-UNESP).

GARCIA, A.J.V. 1991. Evolucão sedimentar da seqüência pré-rift das bacias costeiras e interiores do Nordeste brasileiro. Pesquisas, 18(1):3-12

GHIGNONE, J.I. 1972. Ensaio de paleogeografia do Nordeste e as seqüências sedimentares. In CONGR. BRÁS. GEOL., 26, Belém, 1972. Anais... Belém, SBG, v.3, p. 21-28.

GHIGNONE, J.I. 1979. Geologia dos sedimentos fanerozóicos do Estado da Bahia. In: INDA, H.A.(ed.) Geologia e Recursos Minerais do Estado da Bahia. Salvador, Secr. de Estado das Minas e Energia da Bahia v. 1, p. 24-117 (Série Textos Básicos)

GHIGNONE, J.I. \& COUTO, E.A. \& ASSINE, M.L. 1986. Estratigrafia e estrutura das bacias do Araripe, Iquatu e Rio do Peixe. In: CONGR. BRAS. GEOL., 34, Goiânia, 1986. Anais... Goiânia, SBG, v. l, p. $271-285$.

LANZARINI, W.L. 1994. Paleocorrentes fluviais e eólicas das formações Aliança e Sergi, Bacia do Recôncavo. In: CONGR. BRÁS. GEOL., 38, Camboriú, 1994. B. Resumos Expandidos... Camboriú, SBG, v. 3, p. $305-307$.

LIMA, M.R. 1978. Palinologia da Formação Santana (Cretáceo do Nordeste do Brasil). São Paulo, 335p. (Tese de Doutoramento, IG-USP).

LIMA, M.R. \& COELHO, M.P.C.A. 1987. Estudo palinológico da sondagem estratigráfica de Lagoa do Forno, Bacia do Rio do Peixe,
Cretáceo do Nordeste do Brasil. São Paulo, B. IG-USP, 18:67-83 (Série Científica)

MABESOONE, J.M. \& TINOCO, I.M. 1973. Paleoecology of Aptian Santana Formation (Northeastern Brazil). Palaeogeogr., Palaeoclim., Paleaeocol., 14(2):87-118.

MABESOONE, J.M. \& LIMA, P.J. \& FERREIRA, E.M.D. 1979. Depósitos de cones aluviais antigos ilustrados pelas formações Quixoá e Antenor Navarro (Nordeste do Brasil). In: SIMP. GEOL. NORDESTE, 9, Natal, 1979. Núií...Natal, SBG, p.225-235.

MACHADO, D.L., Jr.; DEHIRA, L.K.; CARNEIRO, C.D.R. \& ALMEIDA, F.F.M. 1989. Reconstruções paleoambientais do Juro-Cretáceo do Nordeste oriental brasileiro. Rev. Bras. Geoc., 19(4):470-485.

MEDEIROS, R.A. 1990. Estratigrafia da Chapada do Araripe - o estado da arte. In: SIMP. SOBRE A BACIA DO ARARIPE E BACIAS INTERIORES DO NORDESTE, 1, 1990, Crato. Atai... Crato, DNPM, p. 43-51.

MENEZES, N.R., P, SANTOS, R.A. \& SOUZA, J.D. 1988.5an/ii Erigida, Folha SC.24-X-C-V, estados da Bahia e Sergipe. Brasília, MME/ DNPM, 144p. (Progr. Levant. Geol. Bás. do Brasil).

MIALL, A.D. 1974. Paleocurrent analysis of alluvial sediments: a discussion of directional variance and vector magnitude. J. Sed. Petrol., 44(4):1174-1185.

MIALL, A.D. 1990. Principles of sedimentary basin analysis. $2^{\mathrm{s}}$ ed. New York, Springer-Verlag, 668p.

OJEDA, H.A. \& FUJITA, A.M. 1974. Bacia Sergipe/Alagoas: geologia regional e perspectivas petrolíferas. In: CONGR. BRAS. GEOL.,28, Aracaju, 1974. Anais... Aracaju, SBG, v.l, p.137-158.

PETRI, S. 1987. Cretaceous paleogeographic map of Brazil. Palaeogeogr., Palaeoclim., Paleoecoi, 59:117-168.

PETRI, S. \& FÚLFARO, V.J. 1983. Geologia do Brasil (Fanerozóico). São Paulo, T. A. Queiroz/EDUSP, $631 \mathrm{p}$.

PONTE, F.C. (coord.) 1972. Análise comparativa da paleogeologia dos litorais atlânticos brasileiro e africano. Salvador, Petrobrás/SETUPBA,20p.(CPEG-IV).

PONTE, F. C. 1992. Sistemas deposicionais na Bacia do Araripe, Nordeste do Brasil. In: SIMP. BACIAS CRETÁCICAS BRASILEIRAS, 2, Rio Claro, 1992. Resumos Expandidos... Rio Claro, UNESP, p.81-84.

PONTE, F. C. 1994a. Extensão paleogeográfica da Bacia do Araripe no Mesocretáceo. In: SIMP. CRETACEO DO BRASIL, 3, Rio Claro, 1994. Boletim ... Rio Claro, UNESP, p. 131-135.

PONTE, F. C. 1994b. A posição estratigráfica do Grupo Lavras da Mangabeira, interior do Ceará. In: SIMP. CRETÁCEO DO BRASIL, 3, Rio Claro, 1994. Boletim... Rio Claro. UNESP, p. 147-151.

PONTE. F. C. Fo \& PONTE, F. C. 1992. Caracterização estratigráfica da Formação Abaiara, Cretáceo inferior da Bacia do Araripe. In: SIMP. BACIAS CRETÁCICAS BRASILEIRAS, 2, Rio Claro, 1992. Resumos Expandidos... Rio Claro, UNESP, p. 61-64.

POTTER, P.E. \& PETTIJOHN, FJ. 1977. Paleocurrent $s$ and basin analysis. $2^{\mathrm{s}}$ ed., Berlin, Springer-Verlag, $425 \mathrm{p}$.

ROLIM, J.L. 1984. Seqüência elástica e carbonática da Serra do Tona, Macururé, Estado da Bahia. Porto Alegre, 155p. (Tese de Doutoramento, IG-UFRGS).

ROLIM, J.L. \& MABESOONE, J.M. 1982. Um modelo de grande rio para as bacias rift do Recôncavo-Tucano-Jatobá (Purbeckiano-Aptiano, Nordeste do Brasil). In: CONGR. BRÂS. GEOL., 32, Salvador, 1982. Anais... Salvador, SBG, v.4, p. 1406-1412.

SANTOS, M.E.M. 1982. Ambiente deposicional da Formação Santana, Chapada do Araripe (PE/PI/CE). In: CONGR. BRÁS. GEOL., 32, Salvador, 1982.Anais... Salvador, SBG, v.4, p. 1412-1426.

SELLEY, R.C. 1982. Introduction to Sedimenlology. 2« ed., Londres, Academic Press, $417 \mathrm{p}$.

TEIXEIRA, A. S., Neto \& OLIVEIRA, J. J. 1985.0 preenchimento do riftvalley na Bacia do Recôncavo. Rev. Bras. Geoc., 15(2):97-102.
MANUSCRITO A822

Recebido em 9 de dezembro de 1994 Revisão do autor em 27 de junho de 1995 Revisão aceita em 11 de setembro de 1995 